\title{
Tunable 4-Channel Ultra-Dense WDM Demultiplexer with III-V Photodiodes Integrated on Silicon-on-Insulator
}

\author{
Peter De Heyn, Steven Verstuyft, Shahram Keyvaninia, Andrea Trita and Dries Van Thourhout \\ Photonics Research Group, Dept. Information Technology, Ghent University - imec, 9000 Gent, Belgium \\ Center for Nano- and Biophotonics (NB-Photonics), Ghent University, 9000 Gent, Belgium \\ Email: Peter.DeHeyn@intec.ugent.be
}

\begin{abstract}
A tunable 4-channel ultra-dense WDM demultiplexer with $0.25 \mathrm{~nm}$ channel spacing is demonstrated with III-V photodiodes integrated on Silicon-on-Insulator using rib waveguides. A possible application is an in-band label extractor for all-optical packet switching.

OCIS codes: (130.7408) Wavelength filtering devices (250.5300) Photonic integrated circuits
\end{abstract}

\section{Introduction}

While Silicon-on-Insulator (SOI) is gaining in popularity as a substrate for photonic ICs, for its dense integration possibilities, low-loss waveguides and its high-yield high-volume CMOS compatibility, it is still out-performed by III-V semiconductor material for active functionalities such as gain and absorption. For absorption, one can choose for CMOS compatible monolithically grown germanium but this has typically a higher dark current then III-V due to the slight lattice mismatch between germanium and silicon. An alternative is to heterogeneously integrate highquality III-V material on top of SOI by means of molecular or adhesive bonding. We have developed a lowtemperature die-to-wafer bonding process with divinyldisiloxane benzocyclobutene (DVS-BCB) as the bonding layer [1]. The fabrication process consists of bonding unprocessed III-V dies (with the eptixial layers down) onto a processed SOI substrate, removal of the III-V substrate, and subsequent wafer-scale processing for the III-V devices with lithographical alignment accuracy to the underlying SOI structures. This approach has resulted in many active applications ranging from lasers [2], amplifiers [3] and detectors [4] using the underlying SOI for compact routing or filter applications.

Following the same approach we developed a 4-channel demultiplexer aiming for a channel spacing of $0.1 \mathrm{~nm}$ or $12.5 \mathrm{GHz}$ which is usually referred to as the ultra-dense wavelength division multiplexing (UDWDM) grid. In this case the demultiplexer is designed as a label extractor for all-optical packet switching [5] where one wants to filter low-speed in-band addresses from a high-speed (160Gbit/s) data signal. This in-band addresses or labels can then be used to decide how to route the incoming data all-optically into the right channel by e.g. converting it to another wavelength [6]. The labels are extracted from the incoming signal using high-quality factor $(\mathrm{Q})$ add-drop microring resonators and detected afterwards by photodetectors. To extract the in-band label effectively and not distort the incoming data, the filter bandwidth needs to be ultra narrow band and have a free spectral range (FSR) which is large enough, e.g. a FSR=5nm for a $160 \mathrm{Gbit} / \mathrm{s}$ modulated signal. This requirement is reached if one uses a microring resonator with a radius of approximately $20 \mu \mathrm{m}$. Due to the high Q-factor, the filter acts as a low-pass filter such that any remaining higher frequencies of the modulated signal are filtered out [5].

With standard single mode waveguides $(450 \mathrm{~nm}$ wide x $220 \mathrm{~nm}$ high), one cannot use the quasi-TE mode since back-scattering originating from the large field overlap with the vertical sidewall roughness will add up coherently in these high-Q filters. This wavelength dependent backscattering induces serious resonance-splitting and will consequently result in filters with an unacceptable high and varying insertion loss [7]. When one is not restricted to small bends (e.g. a microring resonator with a large FSR needs to have a small circumference) one can use two approaches with a smaller field overlap with the vertical sidewall roughness. However, due to the lower confinement bending losses are more important than scattering loss, so these approaches need in general larger bending radii to still enable high-Q resonators. A first candidate is using the lower confined quasi-TM mode of a fully etched strip waveguide (denoted further as "TM-strip"), which has been used to demonstrate an improved Q-factor in comparison with the quasi-TE mode [7]. The second alternative is to use the quasi-TE mode in a partially etched rib waveguide ("TE-rib") to lower the overlap with vertical sidewall roughness, which has been proven to enable highQ all-pass filters [8]. In this paper we investigate both approaches in a 4-channel demultiplexer configuration.

\section{Design and simulation results}

The TM-strip approach requires a waveguide dimension of height $220 \mathrm{~nm}$ and width $500 \mathrm{~nm}$ and the microring is designed to have a gap between the bus and ring waveguide of $900 \mathrm{~nm}$ and a radius of $23 \mu \mathrm{m}$. For the TE-rib approach the waveguide is defined using a partial etch process of $70 \mathrm{~nm}$, the height of the rib is $220 \mathrm{~nm}$ and the width is $600 \mathrm{~nm}$. The coupling length of the racetrack microring is $4 \mu \mathrm{m}$ and the radius is $24 \mu \mathrm{m}$. The gap between bus and 
ring waveguide is $550 \mathrm{~nm}$. All the ports of the ring are tapered to strip waveguides to allow compact guiding of the light. Due to a limited fabrication precision both the quasi-TM strip mode as the quasi-TE rib mode suffer from random resonance shifts, which can be in the order of $1 \mathrm{~nm}$. Therefore one needs to integrate a separate titanium (Ti) heater on top of each microring resonator to tune the resonances to a specific channel spacing with certain crosstalk.

The detector is designed for a maximized responsivity or in other words: efficient coupling and a minimized overlap with the non-contributive absorption of the p-metal contacts which is especially important when one uses the quasi-TM mode. For this reason the p-contact is split in two, such that the overlap is minimized. The height of the InGaAs absorption layer is optimized for quasi-phase matching between the fundamental SOI mode and the detector mode (second order vertical - fundamental horizontal mode) for both polarizations, resulting in an InGaAs height of 700nm (Fig. 1b). This layer is covered with a $100 \mathrm{~nm} \mathrm{InP} \mathrm{spacer} \mathrm{layer} \mathrm{to} \mathrm{confine} \mathrm{the} \mathrm{optical} \mathrm{detector} \mathrm{mode}$ and thus keeping it away from the top electrical contacts. The width of the Si-waveguide is varied between 1.1, 2 and $3 \mu \mathrm{m}$, which is a trade-off between better phase matching and a faster coupling. The width needs to be wide enough to ensure primary coupling to the horizontal fundamental detector mode which has the lowest overlap with the p-metal contacts in comparison with horizontally higher-order detector modes. With a thin BCB layer of 50 to $100 \mathrm{~nm}$ on top of the Si-waveguide, all the light couples evanescently to the photodetector and gets fully absorbed by the InGaAs layer within respectively 10 to $30 \mu \mathrm{m}$. To make sure that all the light is absorbed, the detector length is varied between 18 and $90 \mu \mathrm{m}$ long in a test suite. The detectors terminating the channels of the demultiplexer are $40 \mu \mathrm{m}$ long, have a $3 \mu \mathrm{m}$ p-metal spacing and a Si-waveguide width of $1.1 \mu \mathrm{m}$. With this InGaAs height, the transit time bandwidth limitation is $34 \mathrm{GHz}$ and the capacitance (depleted regime) of a $40 \mu \mathrm{m}$ long detector will be $80 \mathrm{fF}$.

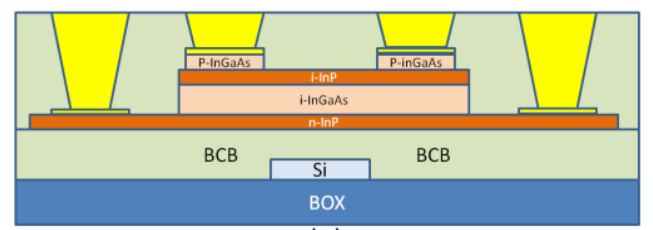

(a)

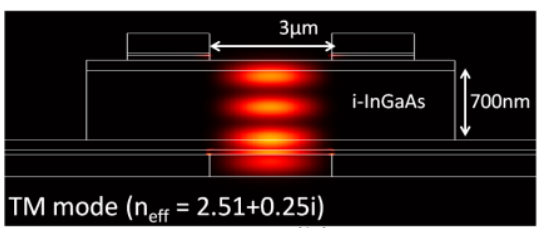

(b)

Fig. 1: (a) Schematic cross section of the photodiode heterogeneously integrated on the Si platform using an adhesive BCB bonding layer (b) Phase matching between the quasi-TM fundamental SOI mode and the III-V photodetector mode

\section{Fabrication}

The UDWDM demultiplexer was fabricated on a 200-mm SOI wafer with $2-\mu \mathrm{m}$ buried oxide and 220-nm top c-Si layer using a subset of processing modules from imec's Silicon-Photonics Platform (iSiPP). Two silicon patterning steps were carried out in which respectively $70 \mathrm{~nm}$ and $220 \mathrm{~nm}$ of the c-Si layer were locally etched to define fibergrating couplers as well as the ring filter and the access waveguides. The III-V photodetector is integrated on the $\mathrm{Si}$ platform using an adhesive bonding technique as mentioned in the introduction. Details can be found in [4,9]. To ensure ohmic contacts, the p-contact consists of a $\mathrm{Ti}(20 \mathrm{~nm}) / \mathrm{Pt}(40 \mathrm{~nm}) / \mathrm{Au}(100 \mathrm{~nm})$ stack deposited on a $50 \mathrm{~nm} \mathrm{p}^{+}-$ type doped InGaAs layer and the n-contact of a $\mathrm{AuGe}(150 \mathrm{~nm}) / \mathrm{Ni}(60 \mathrm{~nm})+\mathrm{Ti}(20 \mathrm{~nm}) / \mathrm{Au}(100 \mathrm{~nm})$ stack deposited on the bottom $50 \mathrm{~nm}$ n-type doped InP layer. The heaters consist of a Ti(120nm)/Au(10nm) stack deposited on a $1.5 \mu \mathrm{m}$ thick BCB layer to avoid optical absorption of the quasi-TM mode. Fig. 2a shows the fully integrated device.

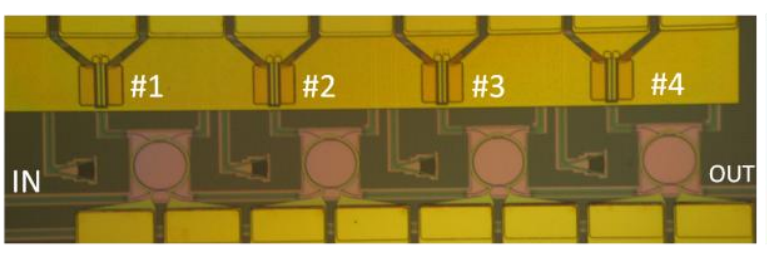

(a)

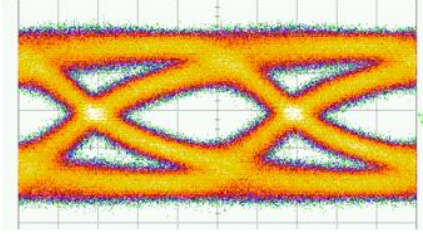

(b)

Fig. 2: (a) Microscopic picture of the 4-channel UDWDM demultiplexer (TE-rib), integrated Ti-heaters and III-V photodiodes (b) 10Gbit/s response of test photodiode of length $40 \mu \mathrm{m}$ (input $1 \mathrm{~mW}$, eye amp. $=14 \mathrm{mV}$, bias $=-3 \mathrm{~V}, \mathrm{~S} / \mathrm{N}$ ratio $=3.6$ ).

\section{Measurement results}

The detector was characterized for both polarizations as a standalone component and in a demultiplexer configuration. The responsivity of the detector is $1.06 \mathrm{~A} / \mathrm{W}$ (quasi-TM) and $1.23 \mathrm{~A} / \mathrm{W}$ (quasi-TE) respectively corresponding to an $85 \%$ and $98 \%$ efficiency for the $18 \mu \mathrm{m}$ long detector (the smallest of the range) with $3 \mu \mathrm{m}$ wide $\mathrm{Si}$-waveguide and does not increase for longer photodiodes. If the Si-waveguide width is decreased to $1.1 \mu \mathrm{m}$, the efficiency is slightly lower for the TE polarization $(1.03 \mathrm{~A} / \mathrm{W})$ due to coupling to higher order modes with a larger 
overlap with the non-contributive metal. The efficiency of the TM-polarized mode could be further increased by increasing the width between the p-contacts (e.g. to $5 \mu \mathrm{m}$ ), but this will increase the capacitance of the photodiode. The dark current of the detector ranges between $3-5 \mathrm{nA}$ for a bias voltage of $-3 \mathrm{~V}$. This bias is needed to work under high power illumination and/or at high speed, otherwise a zero bias is sufficient to reach the maximum responsivity. In Fig. 3Fig. 2b, one can see the large signal response of a 10Gbit/s modulated signal. Estimated bandwidth based on transit time and RC limitation for this $40 \mu \mathrm{m}$ long photodetector is $5 \mathrm{GHz}$ due to the high series resistance $(600 \Omega)$. After rapid thermal annealing (RTA), the series resistance dropped to $130 \Omega$. New measurements are under way but estimations reveal that the bandwidth will be $20 \mathrm{GHz}$, and thus possibly ready for $28 \mathrm{Gbit} / \mathrm{s}$ signals.

The TE-rib approach is characterized on a fully integrated chip (optical to electrical). The filters are tuned to a uniform $0.25 \mathrm{~nm}$ grid with a $-15 \mathrm{~dB}$ XT. The on-chip efficiency is $0.4 \mathrm{~A} / \mathrm{W}$ and the external efficiency of the label extractor is $0.13 \mathrm{~A} / \mathrm{W}$. The heater efficiency for the TE-rib ring resonators is $36 \mathrm{pm} / \mathrm{mW}$ and for this device $9 \mathrm{~mW}$ is needed to tune the heaters on the right grid. The effect of the high-Q low-pass filter in front of the photodetector is currently being investigated.

The TM-strip approach is optically characterized showing very good filter characteristics: a crosstalk on the $0.1 \mathrm{~nm}$ UDWDM grid of $-17 \mathrm{~dB}\left(\mathrm{XT}_{0.1}\right), 20 \mathrm{~dB}$ bandwidth (BW) of $0.3 \mathrm{~nm}$ and an insertion loss (IL) of only $3 \mathrm{~dB}$, which is shown in Fig. 3a. The heaters are performing inefficient $(5.3 \mathrm{pm} / \mathrm{mW})$ because of the low confinement in the Si-waveguide and the thick BCB spacer layer. This is an important drawback of the TM-strip approach but could be resolved using alternative approaches for heater integration. The FSR of the filter (for both approaches) is $5 \mathrm{~nm}$.

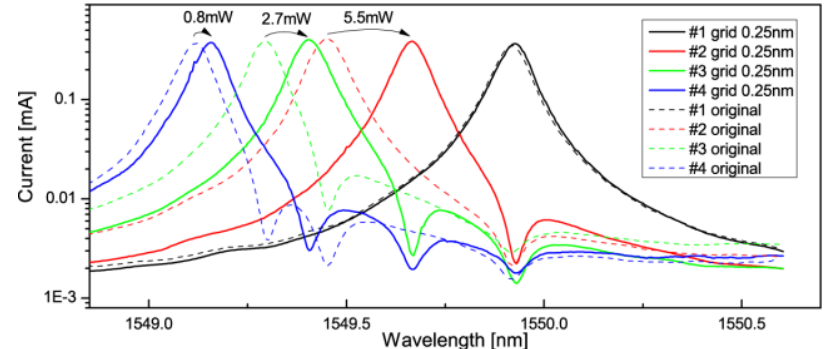

(a)

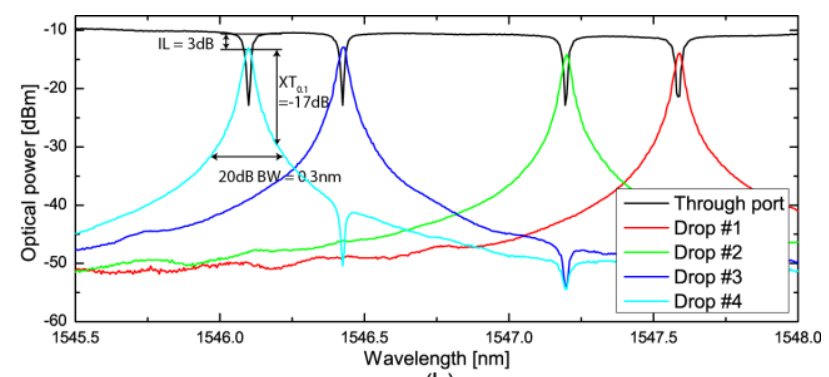

(b)

Fig. 3: The 4-channel demultiplexer (a) optical to optical response of the TM-strip approach (b) the optical-electrical TE-rib approach with channels tuned on a $0.25 \mathrm{~nm}$ grid with $-15 \mathrm{~dB}$ XT

\section{Conclusion}

An ultra-dense WDM demultiplexer with 0.1 and $0.25 \mathrm{~nm}$ channel spacing is designed as a possible in-band label extractor for all-optical packet switching using the TM-polarization with strip waveguides and using the TEpolarization using rib waveguides. A full test employing this functionality is under investigation. The III-V photodetector is heterogeneously bonded on a SOI platform, and has a very good efficiency for both TM and TE polarization, respectively $1.05 \mathrm{~A} / \mathrm{W}$ and $1.25 \mathrm{~A} / \mathrm{W}$ and a very low dark current $(<5 \mathrm{nA}$ for $-3 \mathrm{~V})$. The efficiency for the TM-polarization could be further increased as explained in the paper. The photodetector has a bandwidth of $5 \mathrm{GHz}$ before rapid thermal annealing (RTA) and we believe that this bandwidth could be increased up to $20 \mathrm{GHz}$ after RTA. This makes this photodetector design also suitable for telecommunication applications in need of a polarization independent and fast photodetector, e.g. for dual-polariziation QPSK applications.

6. Acknowledgments: This work is partially supported by the EU project ICT-BOOM and the ERC project ULLPIC

\section{References}

[1] Roelkens, G., et al. (2007). III-V / Si photonics by die-to-wafer bonding. Materials Today, 10(7), 36-43.

[2] Lamponi, M., et al. (2012). Low-Threshold Heterogeneously Integrated InP / SOI Lasers With a Double Adiabatic Taper Coupler. IEEE Photonics Technology Letters, 24(1), 76-78.

[3] Tassaert, M., et al. (2012). An optically pumped nanophotonic InP/InGaAlAs optical amplifier integrated on a SOI waveguide circuit. Optical and Quantum Electronics. Online First ${ }^{\mathrm{TM}}, 8$ March 2012

[4] Sheng, Z., et al. (2010). InGaAs PIN photodetectors integrated on silicon-on-insulator waveguides. Optics express, $18(2), 1756-61$.

[5] Calabretta, N., et al. (2009). All-Optical Label Extractor/Eraser for In-Band Labels and 160-Gb/s Payload Based on Microring Resonators.

IEEE Photonics Technology Letters, 21(9), 560-562.

[6] Stamatiadis, C., et al. (2011). Silicon-on-Insulator Nanowire Resonators for Wavelength Converters, 29(20), 3054-3060.

[7] De Heyn, P., et al (2011). High-Performance Low-Loss Silicon-on-Insulator Microring Resonators using TM-polarized Light. The Optical Fiber Communication Conference and Exposition (OFC) and The National Fiber Optic Engineers Conference (NFOEC) 2011 (p. OThV).

[8] De Heyn, P., et al. (2012). Ultra-high Q and finesse all-pass microring resonators on Silicon-on-Insulator using rib waveguides. (ECIO 2012)

[9] Keyvaninia, S., et al. (2012). Multiple die-to-wafer adhesive bonding for heterogeneous integration. ECIO 2012 (pp. 1-2). 